\title{
Analyses of the short- and long-term graft survival after kidney transplantation in Europe between 1986 and 2015
}

\author{
Maarten Coemans ${ }^{1,2}$, Caner Süsal ${ }^{3}$, Bernd Döhler ${ }^{3}$, Dany Anglicheau ${ }^{4}$, Magali Giral $^{5}$, Oriol Bestard ${ }^{6}$, \\ Christophe Legendre ${ }^{4}$, Marie-Paule Emonds ${ }^{1,7}$, Dirk Kuypers ${ }^{1,8}$, Geert Molenberghs ${ }^{2}$, Geert Verbeke ${ }^{2}$ and \\ Maarten Naesens ${ }^{1,8}$
}

\begin{abstract}
${ }^{1}$ Department of Microbiology and Immunology, KU Leuven, University of Leuven, Leuven, Belgium; ${ }^{2}$ Leuven Biostatistics and Statistical Bioinformatics Centre, Leuven, Belgium; ${ }^{3}$ Institute of Immunology, University of Heidelberg, Heidelberg, Germany; ${ }^{4}$ Service de Néphrologie-Transplantation Adulte, Hôpital Necker, Paris, Université Paris Descartes \& INSERM U 1151, Hôpital Necker, Paris, France; ${ }^{5}$ Centre de Recherche en Transplantation et Immunologie UMR1064, INSERM, Université de Nantes, Nantes, France; ${ }^{6}$ Kidney Transplant Unit, Nephrology Department, Bellvitge University Hospital, Barcelona, Spain; ${ }^{7}$ Histocompatibility and Immunogenetic Laboratory, Red Cross Flanders, Mechelen, Belgium; and ${ }^{8}$ Department of Nephrology and Renal Transplantation, University Hospitals Leuven, Leuven, Belgium
\end{abstract}

The evolution of kidney allograft survival remains insufficiently studied in the context of the changing donor and recipient demographics. Since European data are lacking we performed a cohort study (1986-2015) that, based on the Collaborative Transplant Study, included 108 787 recipients of brain-death kidney donors in 135 hospitals across 21 European countries. We analyzed the hazard rate of kidney failure after transplantation. Between 1986 and 1999, improvement in graft survival was more pronounced in the short term than in the long term: one-, five- and ten-year hazard rates after transplantation declined $64 \%$ (95\% confidence interval, $61 \%-66 \%$ ), 53\% (49\%-57\%) and $45 \%(39 \%-50 \%)$, respectively. Between 2000 and 2015, hazard rates at one, five and ten years posttransplant declined respectively $22 \%(12-30 \%), 47 \%$ (36-56\%) and 64\% (45-76\%). Improvement in graft survival in the first five years post-transplant was significantly less since 2000, while improvement after five years was comparable to before. During the 2000-2015 period improvement of graft survival was greater in the long than in the short term. These changes were independent of changing donor and recipient characteristics, and reflect the evolution in global kidney transplant management over the past decades. Unfortunately, after accounting for the evolution of donor and recipient characteristics, we found that short-term improvement in graft survival decreased since 2000, while long-term improvement remained unchanged in Europe. Thus, deceleration of short-term graft survival improvement in more recent years illustrates an unmet need for innovation.

\footnotetext{
Correspondence: Maarten Naesens, Department of Nephrology and Renal Transplantation, University Hospitals Leuven, Herestraat 49, 3000 Leuven, Belgium.E-mail: maarten.naesens@uzleuven.be
}

Received 14 February 2018; revised 26 April 2018; accepted 17 May 2018
Kidney International (2018) घ, घ-目; https://doi.org/10.1016/ j.kint.2018.05.018

KEYWORDS: nephrology; renal transplantation; survival analysis Copyright (c) 2018, International Society of Nephrology. Published by Elsevier Inc. All rights reserved.

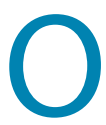
ver the past decades, improvement of graft survival after kidney transplantation has mainly been attributed to better prevention of acute rejection by the introduction of novel immunosuppressive agents such as cyclosporine in the 1980s and mycophenolate mofetil and tacrolimus in the 1990s. ${ }^{1-3}$ Whereas acute rejection is the main barrier for the short-term success of a transplantation, late graft failure is a multifactorial phenomenon. ${ }^{1,2}$

United States registry data illustrated that between 1989 and 2009, graft survival after kidney transplantation mainly improved in the short term, while only very modest improvement was observed in the long term. ${ }^{4-6}$ Because the European and American transplantation reality and survival times differ considerably, with 5-year graft survival rates of $77 \%$ in Europe versus $62 \%$ to $73 \%$ in the United States (US), ${ }^{7,8}$ it remains unclear whether the evolution of allograft survival observed in the US is also valid in Europe.

In this study, we assessed the evolution of kidney survival after transplantation over the past 3 decades in Europe, both in the short and the long term after transplantation, while accounting for the changes in donor and recipient characteristics over time. Indeed, it is well known that the donor and recipient demographics have changed considerably over the past decades: increasingly older donor kidneys are transplanted in increasingly older recipients with increasing comorbidities. ${ }^{9,10}$ While others have examined certain categories of donor and recipient age $^{5}$ or created low- and high-risk (age) groups, ${ }^{4}$ we corrected our analyses for the demographic variables in their most granular form. 


\section{RESULTS}

\section{Demographics}

Of the 108,787 transplantations, 32,956 patients $(30.3 \%)$ experienced kidney failure. Table 1 describes the demographics of our cohort, categorized by decade of year of transplantation. Over the past 3 decades, donors and recipients were getting increasingly older at the time of transplantation (Spearman correlation with decades of 0.37 and 0.28 , respectively; $P<0.001$ for both). This reflects the known evolution of clinical transplantation practice, but also shows the importance of correcting our inferences for these subject-specific characteristics. Supplementary Table S1 describes the use of immunosuppressive regimens across the decades.

\section{Transplantation decade and Kaplan-Meier graft survival}

A Kaplan-Meier survival plot (event $=$ kidney failure after transplantation) analyzed per decade of transplantation is presented in Figure 1. We noticed improvement from the first (1986-1995) to the second (1996-2005) decade, and to a somewhat lesser extent from the second to the last decade (2006-2015). The actual 1- and 5-year death-censored graft survival rates were, respectively, $86.8 \%$ and $74.6 \%$ in patients transplanted between 1986 and $1995,91.1 \%$ and $82.5 \%$ in patients transplanted between 1996 and 2005, and 92.0\% and $84.4 \%$ in patients transplanted between 2006 and 2015 (logrank test $P<0.001)$. However, as this analysis did not take into account other covariates and demographic changes that occurred over time (Table 1), more detailed survival modeling was warranted.

\section{Year of transplantation and the hazard of graft failure}

In order to evaluate the strength of the association between transplant (calendar) year and the hazard of graft failure, we first fitted a simple Cox model, stratified by transplant center. This yielded a highly significant $(P<0.001)$ estimate of -0.03 with a hazard ratio of 0.97 per year $(95 \%$ confidence interval [CI], 0.968-0.972]. This univariate model illustrated that the hazard of graft failure diminished significantly, with $3 \%$ per calendar year, between 1986 and 2015. This implies that the hazard of graft failure would have decreased by $59 \%$ over the 30 -year period of our study. Although this model did not account for other covariates, and assumptions were not thoroughly checked, we used this estimate and its significance as a benchmark for our future models, presented below.

In the multiple model, the effect of transplant year was conditioned on the age of donors and recipients, the rank of transplantation, the number of human leukocyte antigen (HLA) mismatches, and the percentage of panel-reactive antibodies (PRA), parameters that were recorded since the beginning of the study. In Figure 2 the fitted natural cubic spline effects are shown for each independent variable. Graphically, the effect of transplant year suggested a linear decrease of the log hazard ratio of graft failure, with a less pronounced decline since 2000 (Figure 2a). In order to model this nonlinearity, we fitted a broken stick ${ }^{11}$ with a knot at the year 2000. This first multivariate model (Supplementary Table S2) thus illustrated that there was linear improvement in graft survival from 1986 to 1999 , and that there was less, but still significant, improvement between 2000 and 2015 .

Concerning the effect of donor age, we observed that the effect on the (log) hazard of graft failure showed a quadratic trend. Increasing donor age increased the slope of its effect (Figure 2b). Figure 2c shows the nonlinearity of the effect of recipient age: the hazard of graft failure was higher in lower age categories and flattened at approximately 55 years. As suggested by the wide confidence bounds, the increase in log hazard ratio in recipients older than 68 years should not be over-interpreted, so that the effect could be appropriately modeled by using the inverse of recipient age. The number of HLA mismatches and the percentage of PRA showed linear and logarithmic trends, respectively, and were included accordingly (Figure $2 \mathrm{~d}$ and e).

Next, we evaluated the interactions of transplant year with the rank of transplantation, donor and recipient age, and the interaction of donor age with recipient age. All interactions were significant $(P<0.001,=0.001,<0.001$, and $<0.001$, respectively), indicating that the hazard improvement per

Table 1 | Demographics of the kidney transplants from deceased brain-death donors $(N=108,787)$ included in this study

\begin{tabular}{|c|c|c|c|c|}
\hline Decade of year of transplantation & $\begin{array}{c}\text { Total } \\
(n=108,787)\end{array}$ & $\begin{array}{c}1986-1995 \\
(n=42,868)\end{array}$ & $\begin{array}{c}1996-2005 \\
(n=39,538)\end{array}$ & $\begin{array}{c}2006-2015 \\
(n=26,381)\end{array}$ \\
\hline Censored, $n(\%)^{a}$ & $75,831(69.7)$ & $24,644(57.5)$ & $28,516(72.1)$ & $22,671(85.9)$ \\
\hline Female recipient gender, $n(\%)$ & $41,154(37.8)$ & $16,266(37.9)$ & $15,056(38.1)$ & $9832(37.3)$ \\
\hline Female donor gender, $n(\%)$ & $44,386(40.8)$ & $15,482(36.1)$ & $16,751(42.4)$ & $12,153(46.1)$ \\
\hline Recipient age, mean (SD) & $48.07(13.24)$ & $44.05(12.50)$ & $48.64(12.85)$ & $53.74(12.75)$ \\
\hline Donor age, mean (SD) & $45.75(15.55)$ & $39.42(13.89)$ & $47.15(14.83)$ & $53.95(14.80)$ \\
\hline Repeated transplants, $n(\%)$ & $17,143(15.8)$ & $6368(14.9)$ & $6502(16.4)$ & $4273(16.2)$ \\
\hline PRA\%, mean (SD) & $6.09(17.06)$ & $6.91(18.22)$ & $5.74(16.37)$ & $5.26(16.01)$ \\
\hline Time on dialysis in months, mean (SD) & $54.57(52.03)$ & $46.42(46.29)$ & $58.12(55.64)$ & $62.53(53.55)$ \\
\hline $\mathrm{ECD}, n(\%)^{\mathrm{b}}$ & $25,559(24.2)$ & $3664(9.1)$ & $10,251(26.2)$ & $11,644(44.5)$ \\
\hline Cold ischemia time in hours, mean (SD) & $19.13(7.61)$ & $22.57(7.87)$ & $18.21(6.68)$ & $14.67(5.51)$ \\
\hline HLA mismatches, mean (SD) & $2.84(1.43)$ & $2.60(1.31)$ & $2.83(1.44)$ & $3.24(1.52)$ \\
\hline
\end{tabular}

ECD, expanded criteria donor; HLA, human leukocyte antigen; PRA, panel-reactive antibodies.

a Patients were censored at time of loss to follow-up, at time of death with a functioning graft, or at January $24,2017$.

${ }^{b}$ Frequency is based on 105,512 transplantations. 


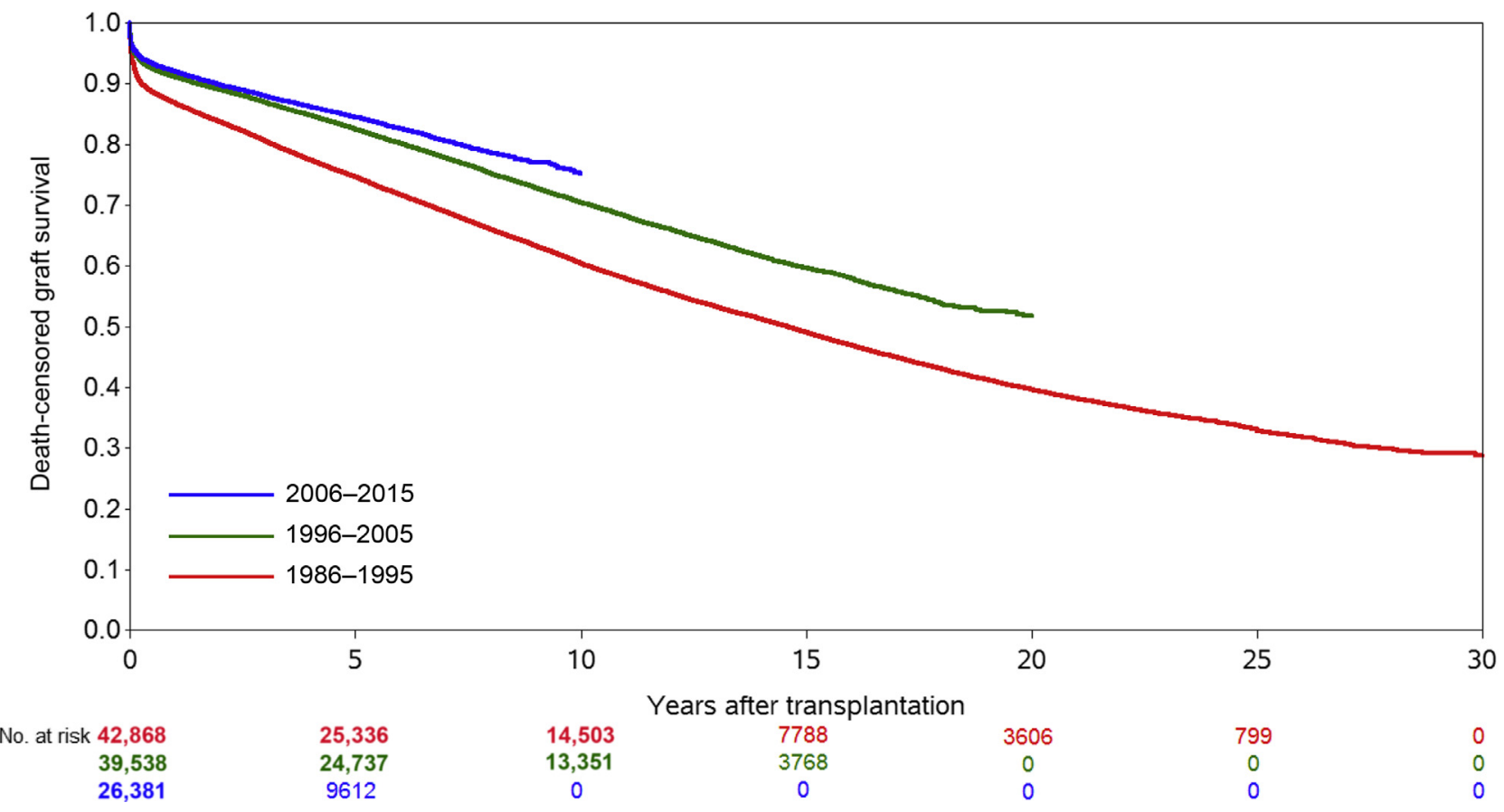

Figure 1 | Kaplan-Meier curve of kidney allograft survival (censored for recipient death), per decade of transplant year.

calendar year partly depended on the age of donors and recipients and on the rank of transplantation. However, as Supplementary Figure S1 shows, these dependencies were very small and did not influence our main conclusions relevantly. Given this and the large power inherent to our data set, we decided to omit these interaction effects from further analyses.

With regard to proportionality, we checked the smoothed plots of the Schoenfeld residuals, provided in Supplementary Figure S2. The plots of transplant year, transplant year slope adjustment since 2000, donor age, and reciprocal recipient age suggested small deviations from proportionality. Hence, we concluded that a flexible time-by-covariate interaction for the first 2 effects and an interaction with the natural logarithm of posttransplant time for the latter ones were needed.

\section{Flexible modeling of the association between transplant year and graft failure}

For the flexible time dependency of the effect of transplant year on graft outcome and its slope adjustment since 2000, the lowest Akaike information criterion, and thus the best fit, was obtained using 5 knots for both effects. This showed that the effect of transplant year on graft outcome, and its linear adjustment since the year 2000, are indeed time-dependent. The functional form of the time dependency (Supplementary Figure S3A) suggested that before 2000 improvement in graft survival has remained constant from 0 to approximately 3 years after transplantation, after which it started decelerating and eventually leveling out from 7 years after transplantation and onward. Since the year 2000, improvement in graft survival has remained constant from
0 to approximately 3 years after transplantation, but then started accelerating until approximately 10 years after transplantation (Supplementary Figure S3B). We also included the possible time dependencies of the effects of donor age and reciprocal recipient age via an interaction with the natural logarithm of time ( $P=0.060$ and $P<0.001$, respectively). As only the interaction with recipient age was significant, we solely withheld this time dependency, leading to the conclusion that the effect of recipient age on graft failure was larger in the long term than in the short term after transplantation.

The final model is presented in Table 2. The parameter estimates of the year of transplantation, its slope adjustment since 2000, and recipient age need to be interpreted together with their time dependencies. Figure 3 and Supplementary Figure S4 illustrate the hazard ratio of graft failure for transplant year, including its time dependency. This showed that during the period of 1986 to 1999, improvement in graft outcome after kidney transplantation was greatest in the early years after transplantation, with less improvement late after transplantation. In addition, this model illustrated that significantly less improvement in graft survival was obtained during the first 5 posttransplant years from 2000 onward, while there was no difference in improvement in the longer term. Thus, between 2000 and 2015, improvement in graft survival per transplant (calendar) year was more pronounced in the long term than in the short term after transplantation.

Translated into concrete examples, the final model illustrated that at 1-year after transplantation, a 64\% (95\% CI, 61\%-66\%) decline of the hazard rate of graft failure was observed from 1986 to 1999. The hazard rate of graft failure at 5 and 10 years after transplantation declined 53\% (95\% CI, 49\%-57\%) and 45\% 

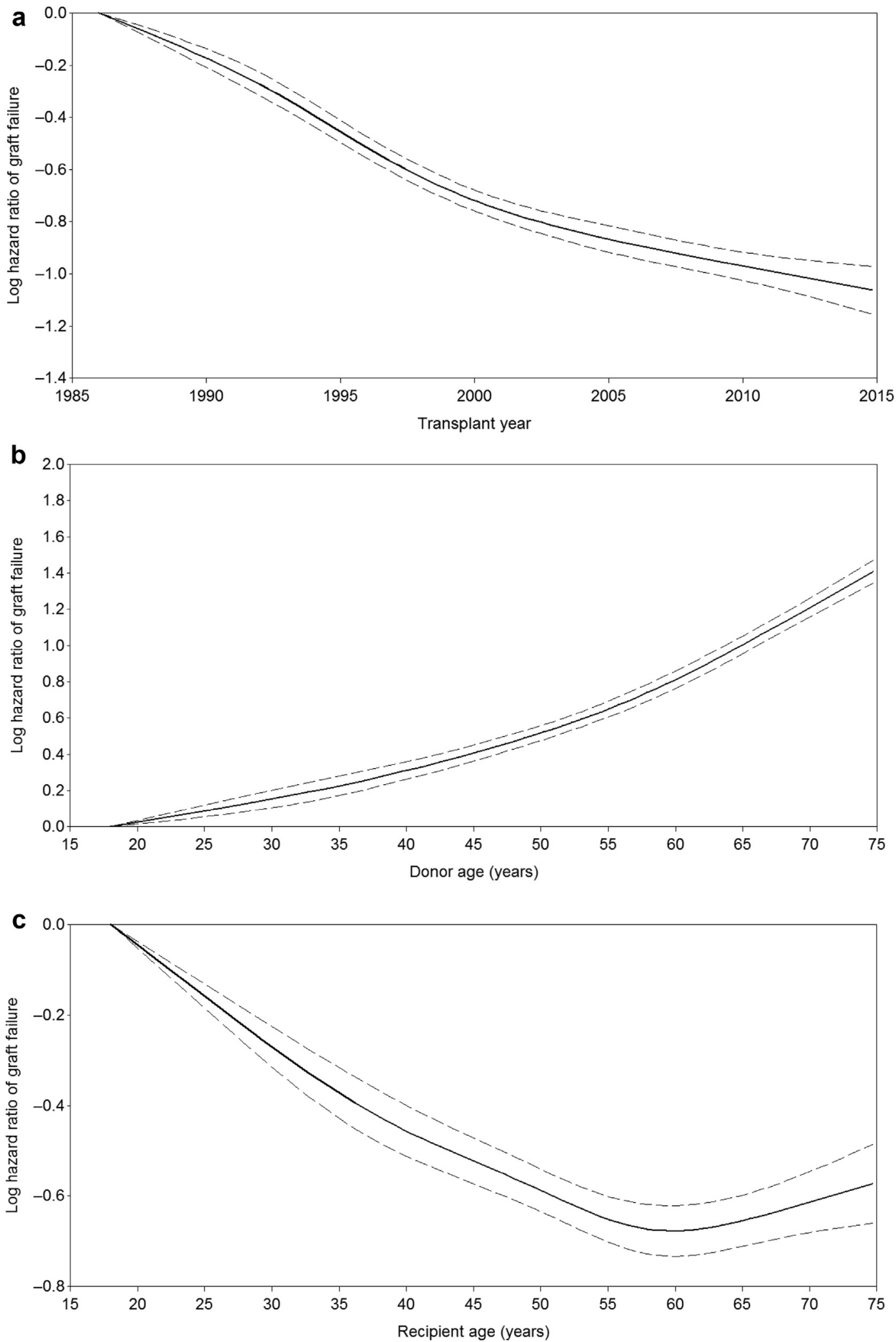

Figure 2 | Effect plot of (a) transplant (calendar) year, (b) donor age, (c) recipient age, (d) human leukocyte antigen (HLA) mismatches, and (e) panel-reactive antibodies (PRA) expressed on the log hazard ratio of graft failure. The log hazard ratio is the natural logarithm of the hazard ratio. The plots show the increase or decrease of the log hazard ratios with increasing covariate values. From this, we were able to find the functional form of the covariate effects. A straight line indicates a linear effect. The plots allowed us to accommodate violated linearity assumptions and should be used for this purpose solely. Log hazard ratios should not be interpreted as an absolute quantity: exponentiation is needed to arrive at the hazard ratios. HLA-A+B+DR mismatches, human leucocyte antigen $A+$ human leucocyte antigen $B+$ human leucocyte antigen DR mismatches. (Continued) 

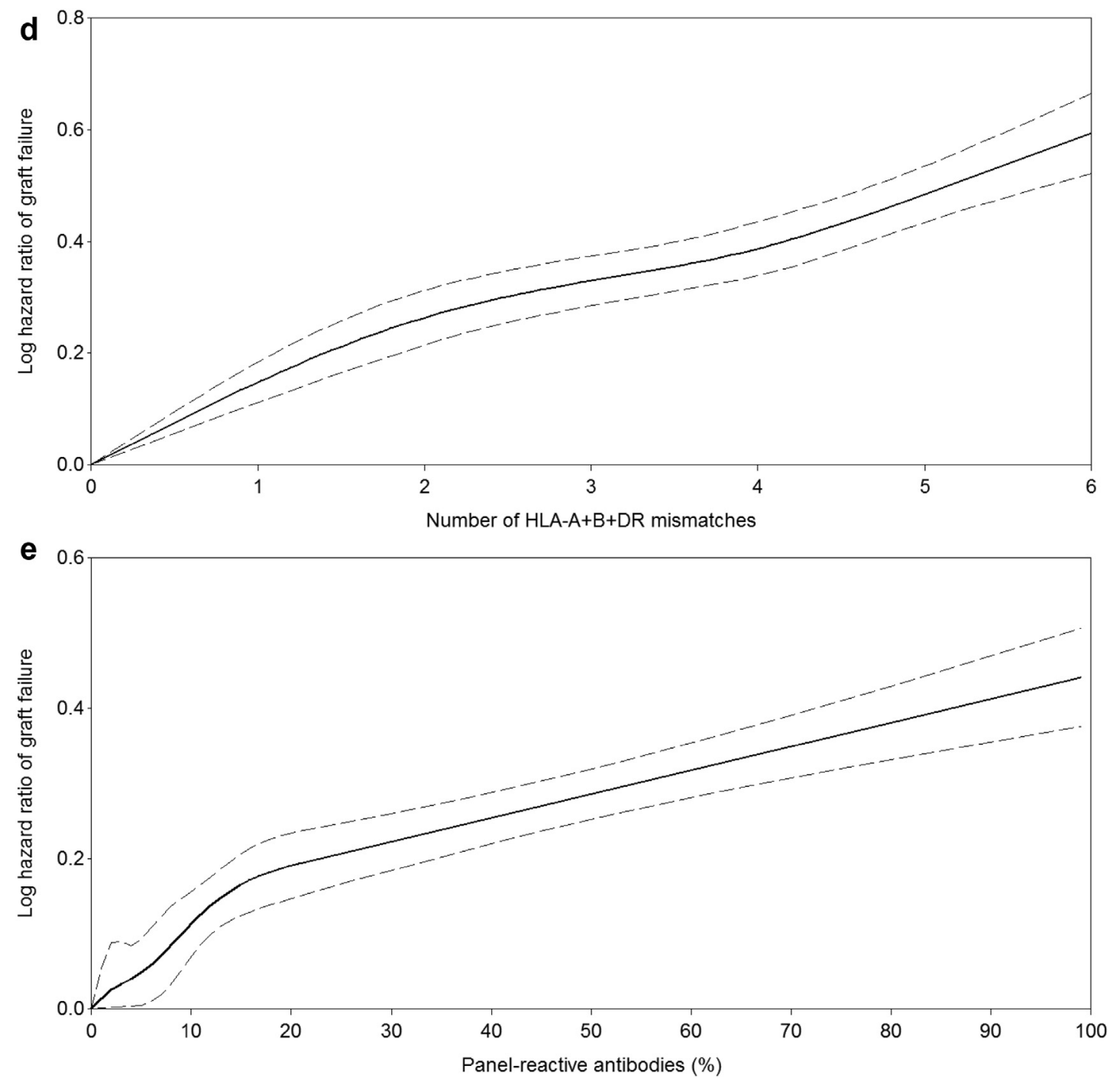

Figure 2 | (Continued).

(95\% CI, 39\%-50\%), respectively, in the same time period (1986-1999). From 2000 to 2015, hazard rates of graft failure at 1 , 5, and 10 years after transplantation declined, respectively, $22 \%$ (95\% CI, 12\%-30\%), 47\% (95\% CI, 36\%-56\%), and 64\% (95\% CI, 45\%-76\%). These conclusions were conditional on donor age, recipient age, transplant rank, number of HLA mismatches, and percentage of PRA.

\section{Sensitivity analysis}

Whereas our conclusions were drawn adjusting for donor age, other aspects of donor organ quality could also be of importance for graft outcome. We therefore performed a sensitivity analysis on the subset of transplantations with expanded-criteria donor kidneys only ( $n=25,559)$. Due to missingness and high collinearity with donor age (Spearman correlation $0.72, P<$ 0.001 ), this parameter could not be included in the main analysis. The sensitivity analysis on the expanded-criteria donor kidney transplants confirmed a similar pattern of improvement of graft outcome over time (Supplementary Figure S5), fully corroborating the aforementioned conclusions. A second sensitivity analysis was performed on an imputed dataset $(n=$ 144,593), assuming missing at random, and including the covariates cold ischemia time, waiting time on dialysis, and cause of donor death (trauma, cerebrovascular accident, or other). Again, the improvement pattern was confirmed (Supplementary Figure S6).

\section{DISCUSSION}

We investigated the evolution of the hazard of kidney transplant failure in Europe over the past 3 decades, taking into account the changes in donor and recipient characteristics that occurred in this period. We had complete data on 108,787 transplantations and analyzed the hazard of losing a kidney after transplantation via a stratified Cox model with a flexible time-dependent effect. By testing and accommodating the 2 underlying assumptions of the Cox model, namely linearity and proportionality, we drew 4 very important conclusions. First, and most importantly, we demonstrated 
Table 2 | Final Cox model for death-censored graft failure

\begin{tabular}{|c|c|c|c|}
\hline \multirow[b]{2}{*}{ Parameters } & \multicolumn{3}{|c|}{ Final model } \\
\hline & Estimates (SE) & $P$ value & $\begin{array}{c}\text { Hazard ratio } \\
\text { (at day } 0 \text { posttransplant }\end{array}$ \\
\hline Transplant year slope adjustment since 2000 & $0.03(0.006)$ & $<0.001$ & 1.03 \\
\hline Slope of year of transplantation since 2000 & ${ }^{-}$ & a & 0.97 \\
\hline Donor age & $-0.002(0.002)$ & $<0.001$ & b \\
\hline Donor age ${ }^{2}$ & $2.70^{*} 10^{-4}\left(2.23^{*} 10^{-5}\right)$ & & $-b$ \\
\hline $\operatorname{Ln}(P R A)$ & $0.07(0.004)$ & $<0.001$ & 1.07 \\
\hline Repeat transplantation & $0.33(0.02)$ & $<0.001$ & 1.39 \\
\hline Transplant year $\times$ spline(time) & $c_{-}^{-}$ & $<0.001$ & ${ }_{-}^{d}$ \\
\hline Transplant year slope adjustment since $2000 \times$ spline(time) & ${ }_{-}^{-} \mathrm{c}$ & $<0.001$ & $-{ }_{-}$ \\
\hline$(1 /$ Recipient age $) \times \ln ($ time $)$ & $6.31(0.65)$ & $<0.001$ & ${ }_{-}^{-}$ \\
\hline
\end{tabular}

HLA-A+B+DR mismatches, human leucocyte antigen A + human leucocyte antigen B + human leucocyte antigen DR mismatches; Ln, natural logarithm; PRA, panel-reactive antibodies.

The model describes the parameter estimates, hazard ratios and their $P$ values after correcting for non-linearity, interactions, and the proportionality assumption.

${ }^{a}$ These values could not be calculated because these estimates were dependent on time after transplantation. The hazard ratio of transplant year is provided graphically in Figure 3.

${ }^{\text {b}}$ These hazard ratios were not calculated because they were dependent on their transformation (quadratic term).

'These values consist of 4 estimates (spline with 5 knots) that cannot be interpreted as such. The hazard ratio of transplant year is provided graphically in Figure 3 .

${ }^{\mathrm{d}}$ Hazard ratios are reported at day 0 posttransplant. Because these terms are used to model the time dependency of the hazard ratios, they are undefined at day 0 .

that there has been improvement in graft outcome during the period of 1986 to 1999 and also since 2000, but significantly less in the second period. Second, although there has been improvement both in the short and in the long term after transplantation, between 1986 and 1999 the latter improved less, which is in concordance with the general observations based on the (American) Scientific Registry of Transplant Recipients database. ${ }^{4-6}$ Third, we found that the cause of decreased improvement since 2000 was mainly due to differences in the short term after transplantation and that, within this period, improvement was larger in the long term. Fourth, the improvement we observed over time before 2000,

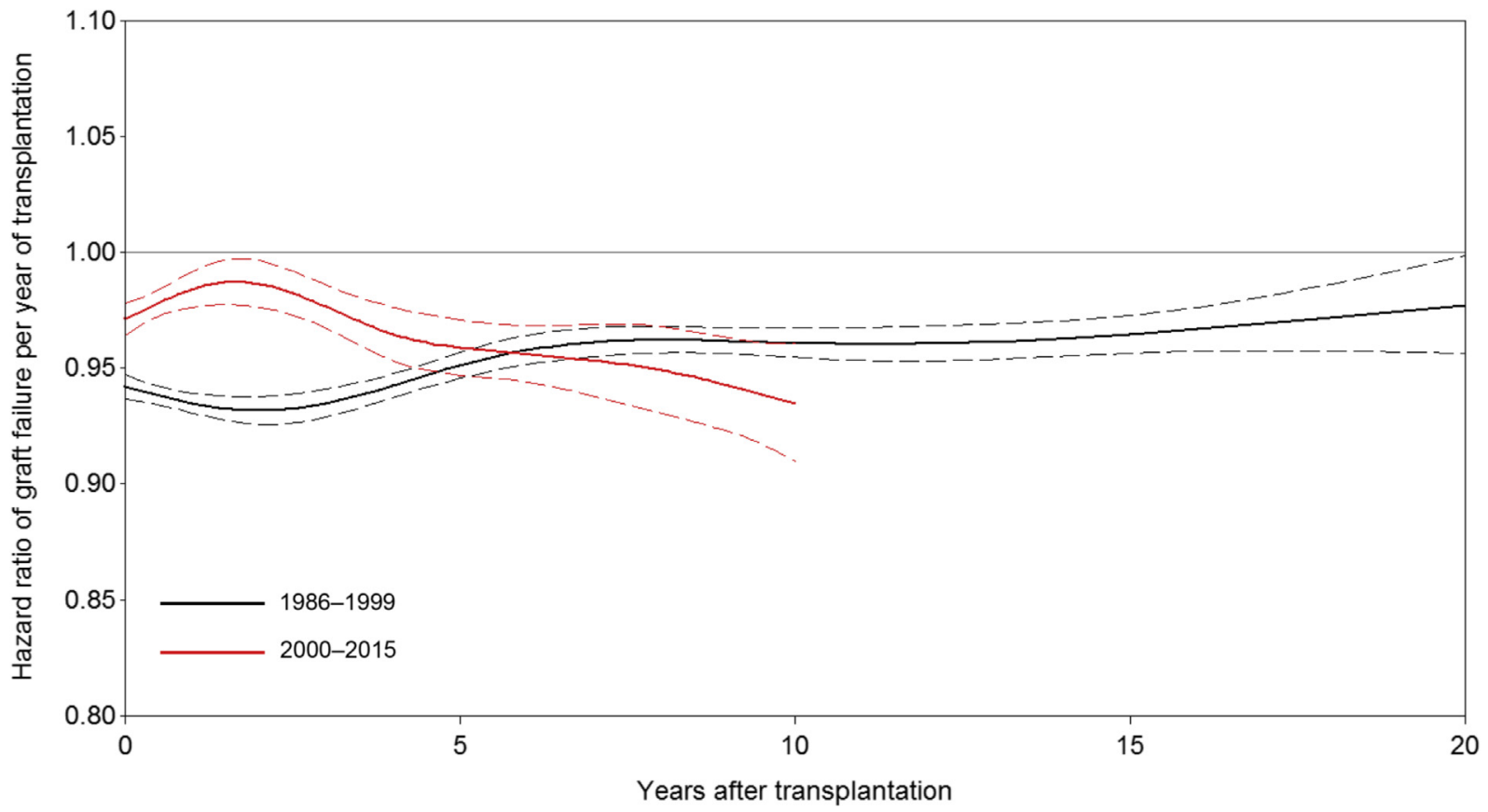

Figure 3 | Hazard ratio of graft failure per transplant (calendar) year, by posttransplantation time, between 1986 and 1999 and between 2000 and 2015. The black line shows the hazard ratio from 1986 to 1999, and the red line shows the hazard ratio from 2000 onward. Before 2000, improvement of graft outcome per transplant (calendar) year mainly occurred in the first 5 years after transplantation, whereas there was significantly less improvement during the first 5 posttransplant years since 2000 . After posttransplantation year 5 , there was no difference in improvement of the hazard of graft failure before 2000 versus after. Data beyond 20 posttransplantation years for the patients transplanted before 2000 and data beyond 10 posttransplantation years for the patients transplanted since 2000 were not provided in this graph due to the increased uncertainty beyond these limits, which is illustrated in Supplementary Figure S4 (which also includes the hazard ratios observed beyond these limits). 
and the lesser improvement since 2000, cannot be attributed to the changing donor and recipient demographics or donor organ quality, but rather reflect the evolutions in global posttransplant management over the past decades.

The deceleration of short-term improvement in graft survival since 2000 is striking and contrasts with the important improvement in graft survival that was made between 1986 and 1999. The main change in clinical kidney transplantation between 1986 and 1999 was the general access to increasingly powerful and innovative immunosuppressive regimens, which translated into decreasing acute rejection rates, ${ }^{1,12,13}$ and is a likely explanation for the large improvement in graft survival during this time frame, despite expansion of the transplantation cohort to older donors and recipients. ${ }^{14}$ Apart from changes in immunosuppression and related decreased risk of acute rejection, other factors such as better control of hypertension, anemia, hypercholesterolemia, and hyperglycemia; more standardized histological evaluation of kidney biopsies; better donor management; improved kidney storage and preservation; updated allocation procedures and (cross-) matching techniques; better detection, prevention, and treatment of infections including polyomavirus nephropathy; better management of cardiovascular and urologic complications; growing team experience; and other measures in kidney transplant management will all have contributed to the improvement of graft outcome before 2000. ${ }^{15-18}$ Which of these factors was most important in the improvement of graft survival cannot be inferred from our data.

In contrast to what is commonly suggested, our study shows that the changing donor and recipient characteristics are not the cause of this deceleration of improvement of graft survival in more recent years. Consequently, the causes for the observed deceleration of improvement in graft survival are to be sought in the factors for which we did not adjust in the analyses. The deceleration of improvement of short-term graft survival since the year 2000 is therefore related to the decrease in improvement in the global management of kidney transplant recipients on all possible fronts, although it is inherently impossible to point to specific factors that lacked innovation. As improvements in global clinical outcome reflect the cumulative impact of many potential contributing factors, deceleration of improvement is the illustration of a global problem, the lack of innovation in global management of kidney transplantation including the lack of better immunosuppressive protocols since 2000. The virtual standstill in the standard immunosuppressive protocols is illustrated by the observation that no novel immunosuppressive regimens have replaced the highly successful combination of tacrolimus and mycophenolate for prevention of acute rejection in standard clinical practice, which was implemented rapidly since the late 1990 s. $^{9}$ Since 2000 , the transplant community has focused on decreasing or eliminating steroids and calcineurin inhibitors, and on the introduction of inhibitors of the mammalian target of rapamycin, but this did not translate into graft survival benefits. ${ }^{19}$ In contrast, many patients lost their grafts under these regimens due to donor-specific antibody development and antibody-mediated rejection. ${ }^{20,21}$ Novel immunosuppressive regimens such as combination therapy with belatacept and mycophenolate seem promising ${ }^{22}$ but are not used routinely in Europe despite European Medicines Agency approval in 2011. As far as we are aware, our results are the first to reflect the lack of new successful standard immunosuppressive regimens, or other innovations in posttransplant management, in the field of kidney transplantation since the late 1990s.

It is unlikely that potential changes in the immunological risk that is taken play a role in the deceleration of graft survival improvement. Better donor-recipient match evaluation and virtual crossmatching are available in more recent years, and our study conclusions were reached after adjustment for HLA mismatches, repeat transplantation, and HLA sensitization, which reflect the general immunological risk. Adjusting for the presence or absence of donor specificity of HLA antibodies was not possible given the lack of information on this parameter in the patients transplanted in earlier decades. Moreover, if we had been able to adjust for the decreased immunological risk assessment over the 3 decades of the study, ${ }^{23}$ it is unlikely that our conclusions would have changed. Residual confounding of unmeasured donor or recipient risk factors such as cardiovascular risk, metabolic alterations, or surgical risks are possible, but could not be accounted for in the analyses given the lack of such data in the Collaborative Transplant Study (CTS) registry.

Our results are also novel in other aspects. First, our data set encompasses 3 complete decades (1986-2015), thus spanning the full history of transplant practice since the beginning of its widespread clinical use (after the introduction of cyclosporine). Second, we were the first to perform these analyses in Europe, which has a very different health care background and different transplant outcomes compared with the US. ${ }^{8,24}$ Third, we used a more detailed methodology than was previously applied to similar data. More specifically, we were able to make inferences conditional on important characteristics and the changing demographics. Our statistical models allowed us to quantify the changes in graft outcome over the past 3 decades and to model its time dependency.

In addition to our conclusions on the improvement of graft outcome after transplantation over the past 3 decades, several other important inferences could be made. Because we modeled the variables donor and recipient age as continuous functions, we were able to describe their effects in full detail. More specifically, we found that the age of the donor was related to the hazard of graft failure in a quadratic way, which means that 1 year of increase in older donors has more impact on transplant outcome than 1 year of increase in younger donor kidneys. Although others have shown that increased donor age is related to an increased risk of graft failure, ${ }^{4,25}$ by fitting the complete function, the quadratic nature of this association provides relevant additional nuance.

Further, we described that the age of the recipient was inversely related to the hazard of graft failure: younger patients were more inclined to graft failure after transplantation 
than were older patients. This effect of recipient age was no longer valid after the age of 55 years; at that point, all recipients' ages were equally likely to experience graft failure. The association between younger recipients and a higher hazard of graft failure could be explained by higher risks of noncompliance at younger ages and by immune senescence of older patients, as has been reported previously. ${ }^{26,27}$

Despite the merits of our analyses, several limitations warrant attention. First, our sample was not random, but rather originated from a database to which centers contributed on a voluntary basis. Due to this, some selection bias could be present. However, the CTS is the largest kidney transplantation database in Europe, and our study included 21 countries, 135 transplant centers, and as many as 108,787 transplantations. Hence, the diversity within and the size of this sample increase the confidence in our conclusions. In addition, the CTS data collection policy and the built-in data quality control procedures (e.g., center-specific census algorithms) ensure excellent rates of data completion for followup data. The follow-up records of the analyzed European data are complete at 1 year after transplant for $97 \%$ of patients and at year 10 for as high as $92 \%$ of patients. ${ }^{28}$ Second, data about graft survival beyond 20 years are sparse (3.3\%). Therefore, conclusions past this threshold can only be drawn with uncertainty. Third, we excluded patients who received kidneys from donors after cardiac death and from live donors, because these donor types were not or rarely used in the early years of the study. This obviates extrapolating our conclusions to these other types of transplantation. In order to draw similar conclusions in such populations, additional studies with sufficiently long follow-up of these other transplant types are needed. Finally, we analyzed death-censored graft survival. Evaluating the effect on the cause-specific hazard rate allowed us to evaluate whether the rate of kidney graft failure after transplantation decreased from 1986 to 2015 in patients who did not experience a prior event, such as death with a functioning graft. Whereas it is possible that death with a functioning graft is a dependent competing risk, this does not invalidate our conclusions made on the cause-specific hazard rate of graft failure. A common misunderstanding is that competing risk analyses correct for competing events. Instead, a competing risk analysis evaluates whether the absolute risk of kidney graft failure after transplantation decreased from 1986 to 2015, which is highly dependent on the improvement in the rate of death with a functioning graft. If, for instance, the rate of death with a functioning graft improved and the graft failure rate remained constant, the absolute risk for graft failure (artificially) increases. ${ }^{29}$ Given our primary aim to study the relative risk of graft failure with year of transplantation, and given the artificial changes in absolute risk of graft failure with changing rates of death over time, such calculation of absolute risks was not justified in this study.

In conclusion, we demonstrated that in Europe between 1986 and 1999, similar to what has been noted in the US, improvement of kidney graft survival occurred primarily in the short term after transplantation and to a lesser extent in the long term. For the first time, however, we were able to demonstrate that the short-term improvement in graft survival decreased since 2000, while the long-term improvement remained stable, when accounting for the evolution in donor and recipient characteristics. The deceleration of short-term graft survival improvement in more recent years illustrates an unmet need for innovation in this field.

\section{METHODS}

\section{Patients}

Our data originated from the CTS database (available at http://www. ctstransplant.org) to which many transplant centers have contributed voluntarily since 1982 . CTS receives active support from more than 400 transplant centers across 42 countries. For our research, we only considered European data. Moreover, we restricted our analyses to single kidney transplantations with adults between January 1, 1986, and December 31, 2015. The CTS database is the largest, most rigorously managed kidney transplantation database in Europe. Due to the lack of a European registry system, no other database is able to provide data on such a timespan and with such completeness. The methodology of CTS has been explained previously in detail. ${ }^{28}$

\section{Data collection and definitions}

We included the transplant centers with available data from 1986 onward, leading to a total number of 135 centers in 21 European countries. Only transplantations with complete data for the following variables were included: transplant year, survival time, censoring time, donor and recipient age, donor and recipient gender, transplantation rank, number of HLA mismatches on A-, B- and DR-loci, and percentage of PRA. In order to make our sample comparable over time, 30,699 kidney transplantations from living donors and 8777 transplantations with donation after cardiac death were excluded. Both transplantation practices are indeed more recent clinical evolutions. ${ }^{30}$ We assumed the cases without missing values to be a completely random subset of the original database. In total, 108,787 transplantations were included. Graft failure was defined as loss of graft function (return to dialysis or repeat transplantation). In case of death with a functioning graft, we censored graft survival at time of death.

\section{Statistical analysis}

The effect of transplant year on posttransplant graft survival was evaluated using the Cox model, ${ }^{31}$ with the log hazard rate of experiencing kidney failure as the dependent variable and transplant (calendar) year as the main independent (continuous) variable. In the analyses, recipients were censored at time of loss to follow-up, at time of death with a functioning graft, or at January 24, 2017 (last update of the database). This effect was corrected for donor and recipient age, repeated transplant or not, percentage of PRA, number of HLA mismatches, and transplant center, all covariates that were available for the complete observation time. Transplant year serves, at least partially, as a proxy for immunosuppressive advancement. Both the functional form and the time dependency (nonproportionality) of the effect of transplant year and the other covariates were graphically explored. More specifically, we checked linearity by fitting each covariate effect as a natural cubic spline with 5 knots and visually assessing its functional form. The flexibility of the spline functions allowed us to detect any deviation from linearity. Moreover, in case of nonlinearity, we assessed the functional relationship between the dependent and independent variables. In order to check proportionality, we used a spline-based regression line on the weighted Schoenfeld residuals. ${ }^{32,33}$ Large 
deviations from a horizontal line were indicative for nonproportionality. We sequentially ${ }^{34}$ accommodated violated linearity assumptions by transforming the covariates and violated proportionality assumptions by including time-by-covariate interactions. For the main time-by-covariate interaction (transplant year), we included time as a natural cubic spline in order to model the time dependency as flexible as possible. Nonproportionality in other covariate effects was handled by including an interaction term with the natural logarithm of posttransplant time. Traditionally, (continuous) time-varying coefficients in the Cox model are handled by including an interaction term with the covariate and some function of time (= time-to-event). Often these functions are time itself or its $\operatorname{logarithm}^{35}$ : the researcher determines the functional form of the time-varying effect beforehand. ${ }^{36}$ Whereas this approach is defendable, it should not be used in contexts in which the functional form itself is of scientific interest, as was the case in our study. Therefore, as proposed by Hess, ${ }^{37}$ we used a natural cubic spline function of time when considering the interaction with transplant year. In our study, the amount of knots (more knots = more flexibility) was allowed to vary, and the Akaike information criterion guided us to parsimony. The mathematical description of the inclusion of a time-dependent coefficient in the Cox model is provided in the Supplementary Methods section. In order to correct for a center effect, we used stratification. ${ }^{38}$ Stratification allows the centers to have a different baseline hazard function while still assuming equality of the regression coefficients (for the other covariates) across strata. Post hoc sensitivity analyses were performed on the subgroup of expanded criteria donors and on an imputed (fully conditional specification ${ }^{39}$ ) data set, including the extra covariates cold ischemia time, waiting time on dialysis, and cause of donor death. All analyses were performed in SAS 9.4. Next to the traditional procedures we also used the RCS macro ${ }^{40}$ (available at http://cemsiis.meduniwien.ac.at/kb/wf/ software/statistische-software/rcs/) for the spline modeling of the covariate effects and the time dependency. We used 2-sided hypothesis tests with a significance level of $5 \%$.

\section{DISCLOSURE}

All the authors declared no competing interests.

\section{ACKNOWLEDGMENTS}

MC, MPE, and MN are funded by the Research Foundation - Flanders (FWO) and the Flanders Agency for Innovation and Entrepreneurship (VLAIO) with a TBM project grant (no. IWT.150199; "TEMPLATE").

The authors thank the centers and their patients for their participation in the Collaborative Transplant Study (www. ctstransplant.org). Without their generous support, this analysis would not have been possible.

\section{AUTHORSHIP}

$M C$ and MN designed the study and wrote the manuscript. MC, GV, and GM performed the statistical analyses. CS and BD provided the CTS database. MG, OB, DK, MPE, MN, DA, and $C L$ were involved in the data collection. All authors read and revised the manuscript.

\section{SUPPLEMENTARY MATERIAL}

\section{Supplementary Methods.}

Table S1. Immunosuppressive medication per decade.

Table S2. Initial Cox model for death-censored graft failure.

Figure S1. Log hazard ratios of graft failure by transplant (calendar) year, showing its interaction with $(\mathbf{A})$ donor and recipient age and with (B) transplant rank.
Figure S2. Schoenfeld residual plot of (A) transplant (calendar) year, (B) transplant year slope adjustment since 2000, (C) donor age, (D) donor age squared, (E) 1/recipient age, (F) number of HLA-A+B+DR mismatches, (G) In(PRA), (H) repeated transplants.

Figure S3. Dependency on time after transplantation of the log hazard ratio of graft failure per transplant (calendar) year $(\mathbf{A})$ before 2000 and (B) since 2000.

Figure S4. Hazard ratio of graft failure per transplant (calendar) year by time after transplantation, between 1986 and 1999 and between 2000 and 2015.

Figure S5. Hazard ratio of graft failure per transplant (calendar) year by time after transplantation, between 1986 and 1999 and between 2000 and 2015, based on the subset of transplantations with expanded-criteria donors only $(n=25,559)$.

Figure S6. Hazard ratio of graft failure per transplant (calendar) year by time after transplantation, between 1986 and 1999 and between 2000 and 2015, based on a model after multiple imputation, including cold ischemia time, waiting time in dialysis, and cause of donor death as additional covariates ( $n=144,593)$.

Supplementary material is linked to the online version of the paper at www.kidney-international.org.

\section{REFERENCES}

1. Hariharan $\mathrm{S}$, Johnson $\mathrm{CP}$, Bresnahan BA, et al. Improved graft survival after renal transplantation in the United States, 1988 to 1996. N Engl J Med. 2000;342:605-612.

2. Sayegh MH, Carpenter CB. Transplantation 50 years later-progress, challenges, and promises. N Engl J Med. 2004;351:2761-2766.

3. Halloran PF. Immunosuppressive drugs for kidney transplantation. N Engl J Med. 2004;351:2715-2729.

4. Lamb KE, Lodhi S, Meier-Kriesche H-U. Long-term renal allograft survival in the United States: a critical reappraisal. Am J Transplant. 2011;11:450-462.

5. Meier-Kriesche HU, Schold JD, Kaplan B. Long-term renal allograft survival: Have we made significant progress or is it time to rethink our analytic and therapeutic strategies? Am J Transplant. 2004;4:1289-1295.

6. Meier-Kriesche HU, Schold JD, Srinivas TR, Kaplan B. Lack of improvement in renal allograft survival despite a marked decrease in acute rejection rates over the most recent era. Am J Transplant. 2004;4:378-383.

7. Ojo AO, Morales JM, González-Molina M, et al. Comparison of the longterm outcomes of kidney transplantation: USA versus Spain. Nephrol Dial Transplant. 2013;28:213-220.

8. Gondos A, Döhler B, Brenner H, Opelz G. Kidney graft survival in Europe and the United States: strikingly different long-term outcomes. Transplantation. 2013;95:267-274.

9. Hart A, Smith JM, Skeans MA, et al. OPTN/SRTR 2015 Annual Data Report: Kidney. Am J Transplant. 2017;17:21-116.

10. Dreyer GJ, Hemke AC, Reinders MEJ, de Fijter JW. Transplanting the elderly: balancing aging with histocompatibility. Transplant Rev. 2015;29: 205-211.

11. Schwarz CJ. Regression - hockey sticks, broken sticks, piecewise, change points. Course notes for beginning and intermediate statistics. Available at: http://www.stat.sfu.ca/ cschwarz/CourseNotes. Accessed November 7, 2016.

12. Naesens M, Thaunat O. BENEFIT of belatacept: kidney transplantation moves forward. Nat Rev Nephrol. 2016;12:261-262.

13. Pascual M, Theruvath $T$, Kawai $T$, et al. Strategies to improve long-term outcomes after renal transplantation. N Engl J Med. 2002;346:580-590.

14. Hourmant M, Garandeau C. L'évolution de la transplantation rénale ces 20 dernières années. Presse Med. 2011;40:1074-1080.

15. Nankivell BJ, Kuypers DR. Diagnosis and prevention of chronic kidney allograft loss. Lancet. 2011;378:1428-1437.

16. Wekerle T, Segev D, Lechler R, Oberbauer R. Strategies for long-term preservation of kidney graft function. Lancet. 2017;389:2152-2162.

17. Neuberger JM, Bechstein WO, Kuypers DRJ, et al. Practical recommendations for long-term management of modifiable risks in kidney and liver transplant recipients. Transplantation. 2017;101:S1-S56.

18. Solez K, Racusen LC. The Banff classification revisited. Kidney Int. 2013;83 201-206.

19. Lim WH, Eris J, Kanellis J, et al. A systematic review of conversion from calcineurin inhibitor to mammalian target of rapamycin inhibitors for 
maintenance immunosuppression in kidney transplant recipients. Am J Transplant. 2014;14:2106-2119.

20. Opelz G, Döhler B. Effect on kidney graft survival of reducing or discontinuing maintenance immunosuppression after the first year posttransplant. Transplantation. 2008;86:371-376.

21. Wiebe C, Gibson IW, Blydt-Hansen TD, et al. Evolution and clinical pathologic correlations of de novo donor-specific HLA antibody post kidney transplant. Am J Transplant. 2012;12:1157-1167.

22. Vincenti F, Rostaing L, Grinyo J, et al. Belatacept and long-term outcomes in kidney transplantation. N Engl J Med. 2016;374:333-343.

23. Nankivell BJ, P'Ng CH, O'Connell PJ, Chapman JR. Calcineurin inhibitor nephrotoxicity through the lens of longitudinal histology. Transplantation. 2016;100:1723-1731.

24. Squires D, Anderson C. U.S. health care from a global perspective: spending, use of services, prices, and health in 13 countries. Issue Brief (Commonw Fund). 2015;15:1-15.

25. Tullius SG, Tran H, Guleria I, et al. The combination of donor and recipient age is critical in determining host immunoresponsiveness and renal transplant outcome. Ann Surg. 2010;252:662-674.

26. Loghman-Adham M. Medication noncompliance in patients with chronic disease: issues in dialysis and renal transplantation. Am J Manag Care. 2003;9:155-171.

27. Seyda M, Quante M, Uehara $H$, et al. Immunosenescence in renal transplantation: a changing balance of innate and adaptive immunity. Curr Opin Organ Transplant. 2015;20:417-423.

28. Opelz G, Döhler B, Ruhenstroth $A$, et al. The collaborative transplant study registry. Transplant Rev. 2013;27:43-45.
29. Wolbers M, Koller MT, Stel VS, et al. Competing risks analyses: objectives and approaches. Eur Heart J. 2014;35:2936-2941.

30. Domínguez-Gil B, Haase-Kromwijk B, Van Leiden H, et al. Current situation of donation after circulatory death in European countries. Transpl Int. 2011;24:676-686.

31. Cox DR. Regression models and life-tables. J R Stat Soc Ser B. 1972;34: 187-220.

32. Liu X. Survival Analysis: Models and Applications. Chichester, UK: John Wiley \& Sons; 2012.

33. Grambsch PM, Therneau TM. Proportional hazards tests and diagnostics based on weighted residuals. Biometrika. 1994;81:515-526.

34. Keele LJ. Semiparametric Regression for the Social Sciences. Chichester, UK: John Wiley \& Sons; 2008.

35. Allison PD. Survival Analysis Using SAS: A Practical Guide. SAS Institute; 2010.

36. Fisher LD, Lin DY. Time-dependent covariates in the Cox proportionalhazards regression model. Annu Rev Public Heal. 1999;20:145-157.

37. Hess KR. Assessing time-by-covariate interactions in proportional hazards regression models using cubic spline functions. Stat Med. 1994;13:1045-1062.

38. Therneau TM, Grambsch PM. Modeling Survival Data: Extending the Cox Model. New York, NY: Springer; 2000.

39. Van Buuren S, Brand JPL, Groothuis-Oudshoorn CGM, Rubin DB. Fully conditional specification in multivariate imputation. J Stat Comput Simul. 2006;76:1049-1064.

40. Heinzl H, Kaider A. Gaining more flexibility in Cox proportional hazards regression models with cubic spline functions. Comput Methods Programs Biomed. 1997:54:201-208. 